\title{
Localization of plasminogen activator in neonatal lung in the presence of hyaline membrane disease
}

\author{
J. T. ROBERTS \\ From the Department of Pathology, Welsh National School of Medicine, Cardiff
}

SYNOPSIS A case is described in which Todd's method (1959) for the histological localization of plasminogen activator was applied to neonatal lung tissue obtained at (unsuccessful) open cardiac massage. This was localized around small pulmonary veins, usually at some distance from the hyaline membrane.

Neonatal hyaline membrane formation was stated by Lieberman (1961) to be significantly associated with inability of lung tissue to activate plasminogen. This has been denied by Ambrus, Weintraub, Dunphy, Dowd, Pickren, Niswander, and Ambrus (1963), who thought one possible explanation of the discrepancy was the lability of the activator. Lung tissue from a child with hyaline membrane disease, immediately it had been pronounced dead after open cardiac massage, was recently obtained in this labatory, and the results of using Todd's method (1959) for histological localization of activator are of interest.

\section{CASE DETAILS}

This was the second pregnancy of a 32-year-old mother. Her first pregnancy ended in abortion. The child was delivered by Caesarian section, because of placenta praevia, two weeks before term, and was intubated immediately. Subsequently she showed classical signs of the respiratory distress syndrome and died at 36 hours following unsuccessful open cardiac massage.

At necropsy she weighed $2,560 \mathrm{~g}$. Hyaline membrane disease with resorption atelectasis was present.

\section{METHOD FOR DETECTING PLASMINOGEN ACTIVATOR}

The freshly obtained lung was immediately snap frozen in isopentane at the temperature of carbon dioxide snow. It was kept at that temperature until cut, eight hours later. Fibrin slides were prepared by clotting bovine fibrinogen, $0.9 \mathrm{~g} / 100 \mathrm{ml}$., in borate buffer, $p \mathrm{H} 8$, with topical thrombin at 25 units $/ \mathrm{ml}$. Control fibrin slides were heated at $85^{\circ} \mathrm{C}$ for half an hour to destroy plasminogen. Frozen sections at 6 to $8 \mu$ were placed on the slides and

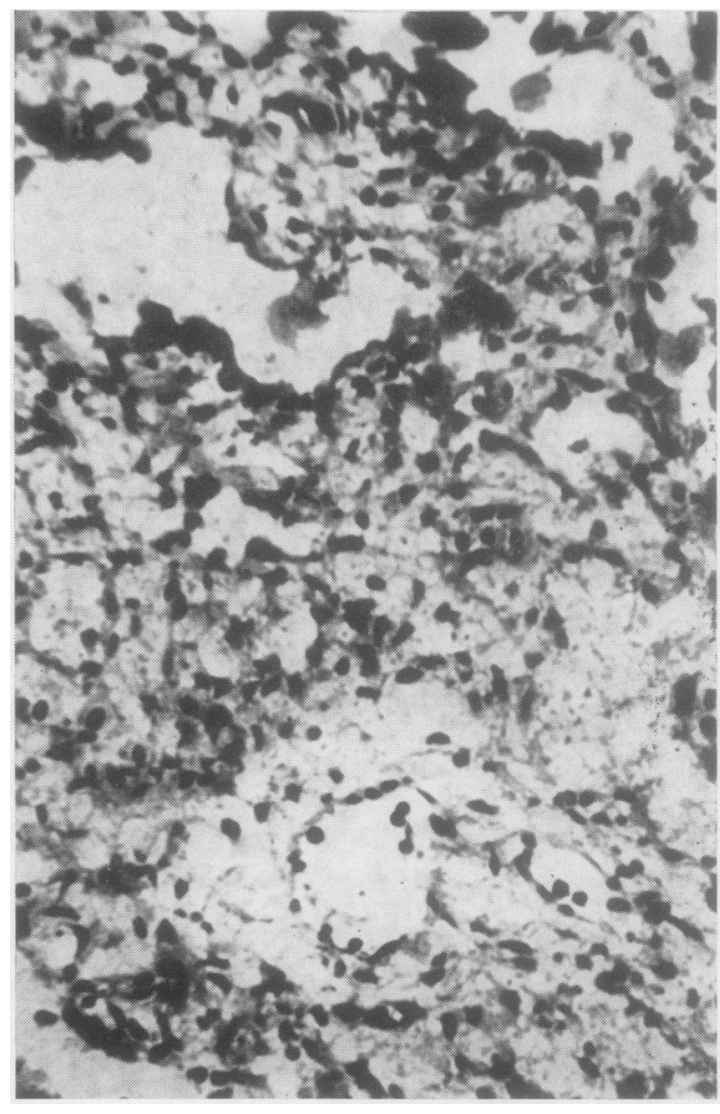

FIG. 1. Frozen section of lung. Hyaline membranes appear as thick black lines and fibrinolysis as a clear area surrounding a vein. Haematoxylin $\times 300$. 
incubated for one and a half hours. Sections were then fixed overnight in formalin vapour and stained with haematoxylin.

\section{RESULTS}

Test sections showed areas of fibrinolysis not present in controls. These surrounded small vessels. In some sites these vessels were unequivocally small veins joining septal veins; elsewhere they were compatible with small veins but identification was uncertain. Lysis was not seen in relation to pulmonary arteries and did not surround the hyaline membranes.

\section{DISCUSSION}

Todd's (1959) method localizes substances that will activate bovine plasminogen to lyse bovine fibrin. Lieberman (1961), who correlated lack of activator with neonatal hyaline membrane formation, used bovine fibrin and assayed homogenized lung. Ambrus et al. (1963), who used products of human origin, found abundant activator in children with hyaline membrane disease. They thought that this difference in assay system, or else a longer period between death and assay, might explain the discrepancy. The present result would favour lability of the activator as the cause.

Todd's work has provided evidence that the source of activator is vascular endothelium, of venules particularly, but in the lung of arterioles also. It is worth observing that the presence of collapse will separate hyaline membranes (with their contained fibrin) from these vascular sources. Adsorption of activator during coagulation appears to be required for the subsequent lysis of fibrin (McFarlane, 1964) and interference with this mechanism might cause fibrin to persist. The fact that blood activator level is high in the neonatal respiratory distress syndrome (Ambrus et al., 1963) need not invalidate this. The concentration at the respiratory surface during the formation of membrane may be more important.

This is an observation on a single case, but there are practical difficulties in obtaining a series and it seemed worth recording. Two neonates without hyaline membrane being formed examined soon after death showed no detectable activator. Both had been on intermittent positive pressure respiration for some hours and were possibly unrepresentative.

I should like to thank Dr. W. B. Shardloe who collected tissue for me and Professor J. Gough for advice. Mr. P. J. Stinchcombe took the photomicrograph.

\section{REFERENCES}

Ambrus, C. M., Weintraub, D. H., Dunphy, D., Dowd, J. E., Pickren, J. W., Niswander, K. R., and Ambrus, J. L. (1963). Pediatrics, $32,10$.

Lieberman, J. (1961). New Engl. J. Med., 265, 363.

McFarlane, R. G. (1964). Brit. med. Bull., 20, 173.

Todd, A. S. (1959). J. Path. Bact., 78, 281. 